\title{
Down-Regulation of USP8 Suppresses HER-3 Positive Gastric Cancer Cells Proliferation
}

This article was published in the following Dove Press journal: OncoTargets and Therapy

\author{
Jiangang Sun ${ }^{1}, *$ \\ Dandan Shen ${ }^{2, *}$ \\ Yongshun Gao' \\ Yichao Zheng $\mathbb{D}^{2}$ \\ Lijuan Zhao ${ }^{2}$ \\ Mamum Maa $\mathbb{( D}^{2}$ \\ Hongmin Liu $^{2}$ \\ Xiaoping Chen ${ }^{1,3}$
}

'Department of Gastrointestinal Surgery, The First Affiliated Hospital of Zhengzhou University, Zhengzhou, Henan, People's Republic of China; ${ }^{2} \mathrm{Key}$ Laboratory of Advanced Pharmaceutical Technology, Zhengzhou University, Zhengzhou, Henan, People's Republic of China; ${ }^{3}$ Department of Hepatic Surgery of Tongji Hospital, Huazhong University of Science and Technology, Wuhan, Hubei, People's Republic of China

*These authors contributed equally to this work
Correspondence: Yongshun Gao Email gaoys@zzu.edu.cn

Yichao Zheng

Email yichaozheng@zzu.edu.cn
Background: Ubiquitin specific peptidase 8 (USP8) has been reported to induce the degradation of several receptor tyrosine kinases such as epidermal growth factor receptor (EGFR), among which human epidermal growth factor receptor-3 (HER-3) is one of them. However, the role and functional mechanisms of USP8 and HER-3 in gastric cancer (GC) remain unknown.

Objective: To explore the function and mechanism of USP8 and HER-3 in the progression of GC.

Materials and Methods: Eighty-eight patients with histologically confirmed GC were recruited for this study. Tumor samples and GC cell lines were used to detect USP8 and HER-3 levels. MGC803 (HER-3 negative GC cell) was selected as the control group and NCI-N87, MKN-45 and AGS (HER-3 positive GC cells) as the experimental group. USP8i and si-RNA were then used to down-regulate USP8 in each group. Apoptosis and cell-cycle experiments were performed to detect the effects of USP8 on GC cells. Cytotoxicity Assay Kit (MTT) and colony formation assays were used to analyze cell proliferation. Cell migration and invasion ability were examined by wound healing. The expression of related mRNA and protein was detected by Western blot and quantitative real-time PCR (qRT-PCR). In vivo experiments were used to examine the effect of USP8 and HER-3.

Results: Patients with high expression of USP8 or HER-3 tumors alone died earlier than those with low expression and the patients with both USP8 and HER-3 high expression had a shorter overall survival than those with the opposite pattern (both USP8 and HER-3 low expression). Down-regulation of USP8 inhibited cell proliferation and cell metastasis and also reduced the HER-3 expression. We also observed that down-regulation of USP8 inhibited the proliferation of GC cells which highly expressed HER-3. Moreover, downregulation of USP8 could promote the apoptosis of HER3-positive GC cells and inhibit the proliferation of them by affecting the cell-cycle. In vivo studies demonstrated that downregulation of USP8 inhibited HER-3 positive tumors growth.

Conclusion: Down-regulation of USP8 inhibits HER-3 positive GC cells proliferation in vivo and in vitro, which indicate that USP8 represents a feasible choice as a therapeutic target for HER-3 positive GC cells.

Keywords: gastric cancer, USP8, HER-3, cell proliferation

\section{Introduction}

$\mathrm{GC}$ is a common malignant tumor in the world, ranking second in the incidence of males and third in females, and more than $60 \%$ of them are in developing countries. $^{1,2}$ The 5-year survival rate of patients with advanced GC is only $5 \%$ $20 \%$. $^{3,4}$ The outcomes are mainly surgery and chemoradiotherapy, but the prognosis of the patients remains poor. ${ }^{5}$ Therefore, it is of great practical significance and 
broad application prospect to strengthen the research of targeted therapy for GC and to seek a better treatment scheme.

These years, a large number of studies have reported that histone modification is closely related to $\mathrm{GC}$. $^{6,7}$ Ubiquitination is one of the most important histone modifications and has been reported to be involved in the progress of GC frequently., ${ }^{8,9}$ As a member of the deubiquitinating enzymes (DUBs) family, USP8 has been proved to be closely related to the occurrence and development of tumors, ${ }^{10,11}$ including breast cancer, ${ }^{12,13}$ lung cancer, ${ }^{14}$ bladder cancer, ${ }^{15}$ cervical cancer, ${ }^{16}$ etc. USP8 was originally identified as a growth regulated ubiquitinspecific protease and is like many other DUBs characterized by its multidomain architecture. ${ }^{17}$ Cleavage of USP8 led to increased deubiquitination of the epidermal growth factor receptor (EGFR), impairing its downregulation and sustaining EGF signaling. ${ }^{18,19}$ It was found that genetic silencing of USP8 led to the downregulation of several receptor tyrosine kinases (RTK) including EGFR, human epidermal growth factor receptor 3 (HER-3) and human epidermal growth factor receptor 2 (HER-2). ${ }^{20}$ HER-3 is a proto-oncogene encodes cell-membrane associated proteins receptor tyrosine kinase 3 (ErbB3), which has been proved to be associated with the prognosis of GC. ${ }^{21}$ However, the relationship between usp8 and gastric cancer has not been studied. Moreover, there is no relevant literature on the combined effect of usp8 and HER3 on gastric cancer. Thus, the relationship between USP8 and HER-3 in GC is worth exploring.

In order to study the relationship between USP8 and tumor, it has been found that its inhibitor DUBs-IN-2 can significantly inhibit the activity of the target (EGFR) of USP8. ${ }^{20,22}$ However, the biological function and molecular mechanisms of USP8 and HER-3 in GC are still unexplored. Here, our data not only identified cooverexpression of USP8 and HER-3 were closely correlated with poor prognosis in patients, but also provided USP8 inhibitor can inhibit proliferation and metastasis of HER-3 positive GC cells significantly in vitro and in vivo.

\section{Materials and Methods GC Samples and Cell Lines}

This study was approved by the Ethics Committee of the First Affiliated Hospital of Zhengzhou University (Approval No. 2019-KY-93), and all patients provided written informed consent and complied with the declaration of Helsinki. Primary GC samples were obtained from 88 patients who were radical resection for GC at the First Affiliated Hospital of Zhengzhou University, China, from August 1, 2011 to June 30, 2014. Each pair of samples consists of cancer and normal tissue from the same patient. Inclusion criteria: (1) Pathological diagnosis of gastric adenocarcinoma and had undergone D2 radical gastrectomy; (2) Pathological stage $\mathrm{T}_{14} \mathrm{~N}_{03} \mathrm{M}_{0}$; (3) Tissue and clinical data were kept intact. Clinical data were followed up by a combination of telephone and medical records. The main end point of the study was the survival time of the patients from the date of surgery to the date of death. The last follow-up was on June 30, 2019. The histological classification of GC was based on the 2019 WHO classification of digestive tumors, ${ }^{23}$ and the clinical staging was based on the TNM staging of $\mathrm{GC}$ in the 8th edition formulated by the American cancer society (AJCC) and the international union against cancer (UICC) in $2017 .^{24}$

The human GC cell lines (MGC-803, NCI-N87, MKN45, AGS, HGC-27 and BGC-823) were purchased from the Shanghai Institutes for Biological Sciences, Chinese Academy of Sciences. All cells were cultured in RPMI1640 (BI, USA) supplemented with 10\% fetal bovine serum (BI, USA) and all cells were incubated in a humidified chamber at $37^{\circ} \mathrm{C}$ with $5 \% \mathrm{CO}_{2}$.

\section{Antibodies and Reagents}

USP8 inhibitor (MB7295), MTT (MA0198) and MG132 (MB4176) were purchased from Meilune (China). AntiUSP8 (ab228572) was purchased from Abcam (USA). Anti-HER-3 (4290S) were purchased from CST (USA). Anti-Ki-67 (AF-0198) was purchased from Affinity (China). Rabbit anti-GAPDH (AB-P-R-001) was purchased from Goodhere (China). And cycloheximide (CHX, 239,763) was obtained from Sigma-Aldrich (USA).

\section{RNA Interference}

Cells were grown in $100 \mathrm{~mm}$ dishes and transfected with USP8-specific siRNA oligonucleotide (Negative control, 5'-UUCUCCAGAACGUGUCACGUTT-3', si-USP8 \#1, 5'-CCACAGATTGATCGTACTAAATT-3' and \#2, 5'TGAAATACGTGACTGTTTA-3', GenePharma) using Lipofectamine 3000 (Invitrogen) according to the manufacturer's instructions. And to confirm knockdown and analyze signaling, cells transfected with si-USP8 were harvested for protein extraction and immunoblotted after 48 to 72 hours. 


\section{RNA Extraction and qRT-PCR}

Total RNA was extracted from trizol reagent according to the manufacturer's instructions (Vazyme, China), and then cDNA was synthesized using PrimeScript RT Master Mix kit (QIAGEN, Germany). The expression of target genes was standardized to the expression of the housekeeping gene glyceraldehyde-3-phosphate dehydrogenase (GAP $\mathrm{DH})$. And the following primers were used to detect the expression of HER-3 and GAPDH: HER-3 forward, 5'AAGCTCTACGAGAGGTGTGA-3', reverse, 5'-TGGGC AATGGTAGAGTAGAG-3'. GAPDH: forward 5'-GATGA CTACCGTCCACTCC-3' and reverse 5'-ACTCTGAAAG CCATACCG-3'.

\section{Cell Viability Assay}

Cell viability was determined using the MTT Cell Proliferation and Cytotoxicity Assay Kit (MTT) following the manufacturer's instructions. $4 \times 10^{3}$ cells were plated in 96-well plates (NEST, China) in $100 \mathrm{~mL}$ volume of media. After incubating in the incubator for 72 hours, add $20 \mu \mathrm{L}$ MTT per well to a final concentration of $5 \mu \mathrm{g} / \mathrm{mL}$, incubate for 4 hours at $37^{\circ} \mathrm{C}$, add $150 \mu \mathrm{L}$ Dimethyl sulfoxide (DMSO, Solarbio, China) to each well and shake for $10 \mathrm{~min}$. Absorbance was measured using microplates reader at a wavelength of $570 \mathrm{~nm}$.

\section{Colony Formation Assay}

In RPMI-1640 medium, 800 cells/well of human GC (MGC-803, AGS and MKN-45) were inoculated into 6-well plates for about 2 weeks. The cells were then washed and fixed with $10 \%$ formalin and stained with a solution of $0.1 \%$ crystal violet (C8470, Solarbio, China). After that, placed them under a microscope for observation.

\section{Western Blot Analysis}

After cell culture meets the requirements, total protein was extracted using radio immunoprecipitation assay (RIPA, Solarbio, China) lysis buffer. Then measured with bicinchoninic acid assays (Solarbio, China) with bovine serum albumin as a standard. Gel electrophoresis was then performed, 5\% milk was incubated for $2 \mathrm{~h}$ and the primary antibody was incubated at $4{ }^{\circ} \mathrm{C}$ overnight. The second antibody was incubated for $2 \mathrm{~h}$ at room temperature and then the membrane was imaged using enhanced chemiluminescence reagent (Thermo, China) and X-ray film (Carestream, China).

\section{Apoptosis and Cell-Cycle Analysis}

GC Cells were planted in 12-well plates and serum-starved overnight. Apoptosis was detected with Annexin V-PE staining kit (Beyotime, China). For cell-cycle, the cells were first starved in serum for 24 hours and then stimulated in complete medium culture for 4 to $12 \mathrm{~h}$. The cell-cycle distribution was evaluated by flow cytometry after staining with sodium propidium iodide (PI, Solarbio, China).

\section{Immunohistochemical Analysis and Evaluation}

Tissues were subjected to immunohistochemical staining (IHC) using specific antibody for USP8 (1:1000), HER-3 (1:1000). Staining was then performed using the diaminobenzidine (DAB kit) (Zsbio, China). Negative control experiments were routinely performed without incubated with specific antibody for USP8 and HER-3. The slices were placed into Aperio AT2 (Leica, USA) for scanning. Aperio image analysis (Leica, Germany) software was used to analyze and establish the scoring template (IHC score). USP8 was located in cytoplasm and cytoplasmic template score was used. HER-3 was mainly located in the cell membrane and was scored by cell membrane template. Cut off line: After ranking the scores from high to low, the first $25 \%$ ( 22 cases) of USP8 was high expression, while the remaining 66 cases were low expression. The first $30.6 \%$ (27 cases) of HER-3 was high expression, while the remaining 61 cases were low expression.

\section{Animal and Xenograft Model}

BALB/c-nu mice (4 weeks old, male) were purchased from Hunan SJA Laboratory Animal Co., Ltd. All of them had adapted for a week and had free access to food and water before the study began. Normal and si-USP8 cells (NCI-N87, $1 \times 10^{7}$ cells in $100 \mu \mathrm{L}$ PBS, MGC-803, $5 \times 106$ cells in $100 \mu \mathrm{L}$ PBS and MKN-45, $5 \times 106$ cells in $100 \mu \mathrm{L}$ PBS) were injected into the hind flank of the nude mice. Each cell line was divided into three groups, control group, USP8 inhibitor group ( $2 \mathrm{mg} / \mathrm{kg}$ USP8 inhibitor for all of them) and si-USP8 group. The USP8 inhibitor was administered intraperitoneally 5 days a week and tumor volume and mice weight were measured every 2 days. Tumor weight was measured after excision on the final day of the experiment. Partial tumor tissues were fixed in formalin, embedded in paraffin, and analyzed by immunohistochemistry after all mice were sacrificed. 


\section{Statistical Analysis}

SPSS 22.0 software (IBM, New York, Armonk, USA) was used to analyze the collected data. The results obtained from cell line experiments and animal assays were analyzed using Student's $t$-test (for two groups) or ANOVA (for more than two groups). The paired $t$-test was used to compare the scores of tumor tissues and normal tissues. Chi-square test was used to analyze the association of the expression of USP8 and HER-3 with clinicopathologic features. Survival analysis was conducted using the Kaplan-Meier survival and Cox proportional hazards model analysis. Data are presented as the mean $\pm \mathrm{SD} . P<0.05$ was considered significant.

\section{Results}

\section{Expression of USP8 and HER-3 and the} Association with Prognosis in GC Patients

To determine whether USP8 and HER-3 were associated with GC, we evaluated the expression pattern of them in cancerous
A

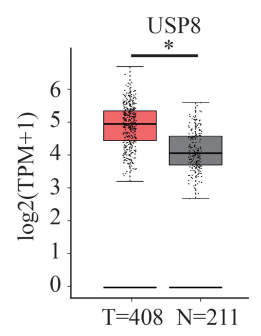

B

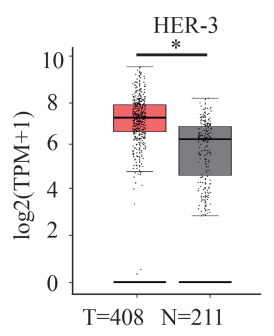

C

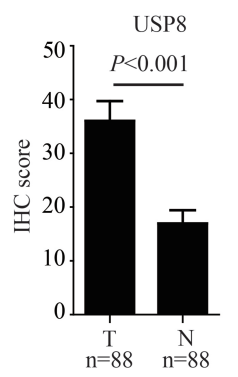

D

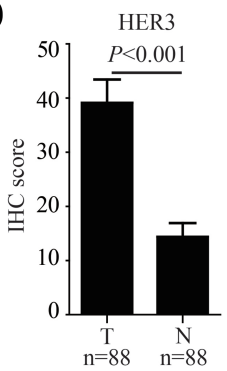

$\mathbf{E}$

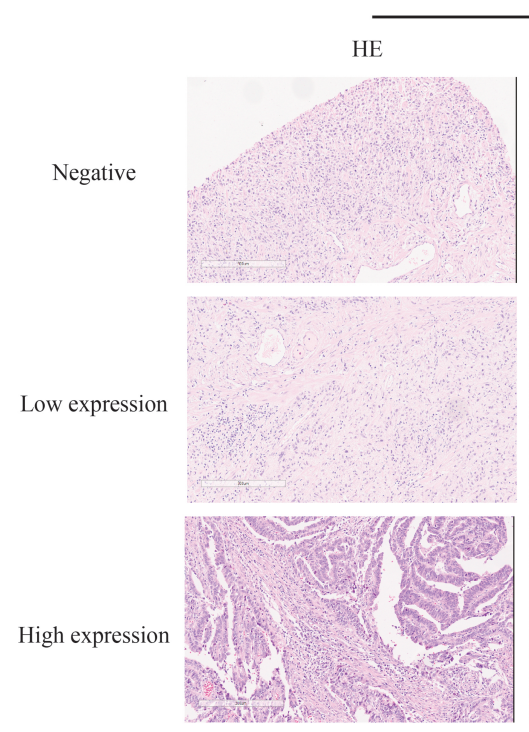

USP8

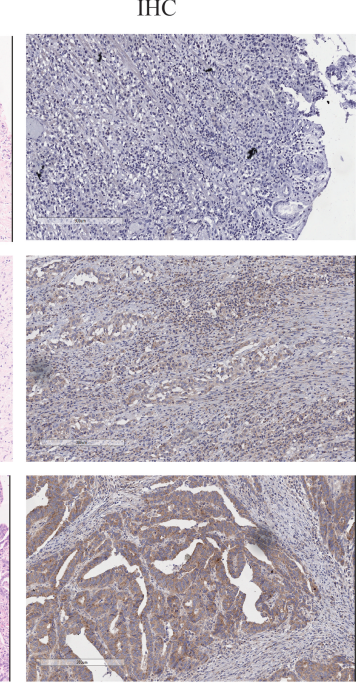

HER-3

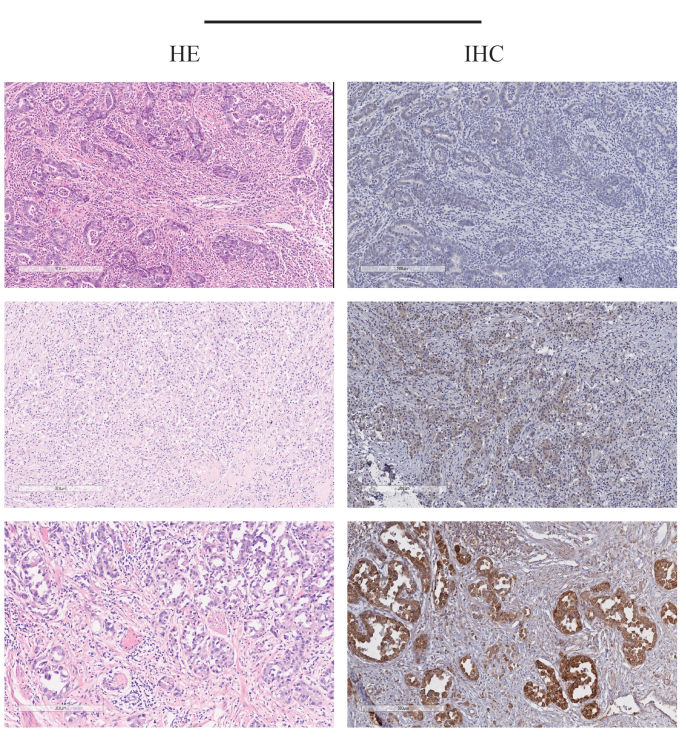

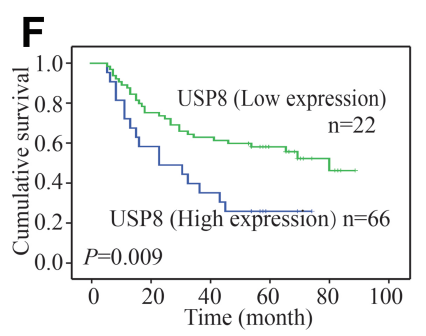
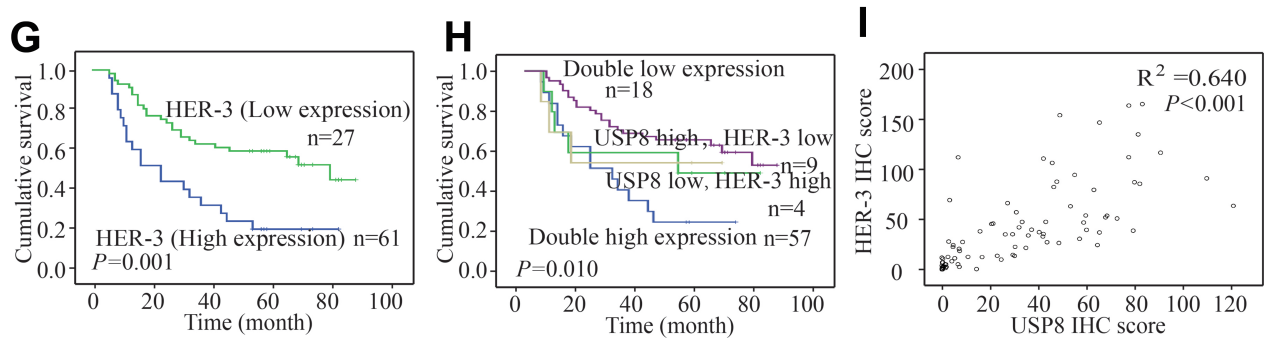

Figure I The expression of USP8 and HER-3 and the association with prognosis in gastric cancer patients (A) As analyzed by gene expression profiling interactive analysis (GEPIA), the USP8 expression level in tumor (T) tissues was significantly higher than nontumor $(\mathrm{N})$ tissues. $* P<0.05$. (B) Analyzed by GEPIA, the HER-3 expression level in tumor $(\mathrm{T})$ tissues was significantly higher than nontumor $(\mathrm{N})$ tissues. $* P<0.05$. (C) Analyzed by immunohistochemical staining (IHC), the USP8 expression level in tumor $(\mathrm{T})$ tissues was significantly higher than nontumor (N) tissues. (D) Analyzed by IHC, the HER-3 expression level in tumor (T) tissues was significantly higher than nontumor (N) tissues. (E) The expressions of USP8 and HER-3 in gastric cancer tissues were evaluated by IHC and hematoxylin-eosin staining (HE, scale bars, $300 \mu$ m. Kaplan-Meier survival analysis of overall survival in all patients according to USP8 (F), HER-3 (G) and USP8/HER-3 (H) expression. The Log rank test was used to calculate $P$ values. (I) Linear regression analysis was used to examine the correlation between USP8 and HER-3 IHC scores levels. $n=88$. 
Table I Expression of USP8 and HER-2 and Patient Characteristics

\begin{tabular}{|c|c|c|c|c|c|c|c|}
\hline \multirow[t]{2}{*}{ Characteristic } & \multirow[t]{2}{*}{ No. } & \multicolumn{2}{|l|}{ USP8 } & \multirow[t]{2}{*}{$P$ value } & \multicolumn{2}{|l|}{ HER-3 } & \multirow[t]{2}{*}{$P$ value } \\
\hline & & High Expression & Low Expression & & High Expression & Low Expression & \\
\hline Gender & & & & 0.862 & & & 0.520 \\
\hline Male & 75 & 19 & 56 & & 24 & 51 & \\
\hline Female & 13 & 3 & 10 & & 3 & 10 & \\
\hline Age $(y r)$ & & & & 0.218 & & & 0.682 \\
\hline$<60$ & 42 & 8 & 34 & & 12 & 30 & \\
\hline$\geq 60$ & 46 & 14 & 32 & & 15 & 31 & \\
\hline Tumor size $(\mathrm{cm})$ & & & & 0.177 & & & 0.991 \\
\hline$<3$ & 27 & 4 & 22 & & 8 & 18 & \\
\hline$\geq 3$ & 61 & 18 & 44 & & 19 & 34 & \\
\hline Location & & & & 0.805 & & & 0.958 \\
\hline AEG & 46 & 11 & 35 & & 14 & 32 & \\
\hline Others & 42 & 11 & 31 & & 13 & 29 & \\
\hline Differentiation & & & & 0.665 & & & 0.810 \\
\hline Well/Moderate & 21 & 6 & 15 & & 6 & 15 & \\
\hline Poor & 67 & 16 & 51 & & 21 & 46 & \\
\hline Lauren type & & & & 0.771 & & & 0.464 \\
\hline Intestinal & 41 & 11 & 30 & & 11 & 30 & \\
\hline Diffuse & 47 & 11 & 36 & & 16 & 31 & \\
\hline Nerve invasion & & & & 0.065 & & & 0.016 \\
\hline Yes & 45 & 15 & 30 & & 19 & 26 & \\
\hline No & 43 & 7 & 36 & & 8 & 35 & \\
\hline Vascular tumor emboli & & & & 0.268 & & & 0.078 \\
\hline Yes & 43 & 13 & 30 & & 17 & 26 & \\
\hline No & 45 & 9 & 36 & & 10 & 35 & \\
\hline T stage & & & & 0.295 & & & 0.112 \\
\hline $\mathrm{TI}+\mathrm{T} 2$ & 19 & 3 & 16 & & 3 & 16 & \\
\hline $\mathrm{T} 3+\mathrm{T} 4$ & 69 & 19 & 50 & & 24 & 45 & \\
\hline$N$ stage & & & & 0.006 & & & 0.001 \\
\hline No & 29 & 2 & 27 & & 2 & 27 & \\
\hline $\mathrm{NI3}$ & 59 & 20 & 39 & & 25 & 34 & \\
\hline pTNM stage & & & & 0.004 & & & 0.021 \\
\hline $1+||$ & 39 & 4 & 35 & & 7 & 32 & \\
\hline III & 49 & 18 & 31 & & 20 & 29 & \\
\hline
\end{tabular}

Note: $P$ values were calculated by the chi-square test.

Abbreviations: AEG, adenocarcinomas of the esophagogastric junction; T, tumor; N, node; PTNM, pathological tumour node metastases.

Table 2 Expression Correlation of USP8 and HER-3

\begin{tabular}{|l|l|l|l|}
\hline \multirow{2}{*}{ USP8 Expression } & \multicolumn{2}{|l|}{ HER-3 Expression } & \multirow{2}{*}{ Total } \\
\cline { 2 - 3 } & High & Low & \\
\hline High & 57 & 9 & 66 \\
Low & 4 & 18 & 22 \\
Total & 61 & 27 & 88 \\
\hline
\end{tabular}

tissue and para-cancer tissue of GC from the gene expression profiling interactive analysis (GEPIA) database (http://gepia. cancer-pku.cn). ${ }^{25}$ From the results in Figure $1 \mathrm{~A}$ and B, the expression of USP8 and HER-3 in cancerous tissue was higher than that in para-cancer tissue. This was consistent with the IHC scores we performed on patient tissue samples, as shown in Figure $1 \mathrm{C}$ and D. To examine the potential effect 
Table 3 Univariate and Multivariate Analyses Comparing Overall Survival to Prognostic Factors in 88 GC Patients

\begin{tabular}{|c|c|c|c|c|}
\hline \multirow{4}{*}{$\begin{array}{c}\text { Factors } \\
\text { Gender } \\
\text { Male } \\
\text { Female }\end{array}$} & \multicolumn{2}{|l|}{ Univariate Analyses } & \multicolumn{2}{|l|}{ Multivariate Analyses } \\
\hline & Relative risk $(95 \% \mathrm{Cl})$ & $P$ value & Relative risk $(95 \% \mathrm{Cl})$ & $P$ value \\
\hline & 1 & 0.356 & & \\
\hline & $1.436(0.667-3.092)$ & & & \\
\hline Age (yr) & & 0.054 & & \\
\hline$<60$ & 1 & & & \\
\hline$\geq 60$ & I.794 (0.99I-3.248) & & & \\
\hline Tumor size $(\mathrm{cm})$ & & 0.046 & & 0.147 \\
\hline$<3$ & I & & I & \\
\hline$\geq 3$ & $2.063(1.137-3.744)$ & & I.86I (0.920-3.766) & \\
\hline Location & & 0.691 & & \\
\hline AEG & I & & & \\
\hline Others & I.I25 (0.630-2.009) & & & \\
\hline Differentiation & & 0.136 & & \\
\hline Well/Moderate & I & & & \\
\hline Poor & $1.787(0.832-3.836)$ & & & \\
\hline Lauren type & & 0.578 & & \\
\hline Intestinal & 1 & & & \\
\hline Diffuse & $0.849(0.475-1.515)$ & & & \\
\hline Nerve invasion & & $<0.001$ & & 0.940 \\
\hline No & I & & 1 & \\
\hline Yes & $15.180(6.911-33.339)$ & & $0.947(0.284-3.153)$ & \\
\hline Vascular tumor emboli & & $<0.001$ & & 0.196 \\
\hline No & I & & I & \\
\hline Yes & $13.215(6.372-27.404)$ & & $2.292(0.798-6.588)$ & \\
\hline T stage & & 0.003 & & 0.659 \\
\hline $\mathrm{TI}+\mathrm{T} 2$ & I & & I & \\
\hline $\mathrm{T} 3+\mathrm{T} 4$ & $5.867(2.180-15.789)$ & & $1.449(0.363-5.790)$ & \\
\hline $\mathrm{N}$ stage & & $<0.001$ & & 0.427 \\
\hline No & 1 & & 1 & \\
\hline NI3 & II.686 (4.362-31.309) & & $0.515(0.130-2.033)$ & \\
\hline pTNM stage & & $<0.001$ & & $<0.001$ \\
\hline I+II & 1 & & I & \\
\hline III & $86.525(16.291-459.549)$ & & $95.012(|2.408-727.53|)$ & \\
\hline USP8 expression & & 0.011 & & 0.037 \\
\hline Low & I & & I & \\
\hline High & $2.220(1.325-3.719)$ & & $3.026(1.264-7.244)$ & \\
\hline HER-3 expression & & 0.002 & & 0.003 \\
\hline Low & 1 & & I & \\
\hline High & $2.516(1.536-4.119)$ & & $4.690(1.993-11.036)$ & \\
\hline
\end{tabular}

of USP8 and HER-3 in GC progression, we measured its expression in GC and adjacent control tissues by hematoxylin-eosin staining (HE) and IHC (Figure 1E). The expression levels of the two proteins were compared with the tumor characteristics and risk factors (Table 1). And the following analysis showed that $\mathrm{N}$ stage, and pTNM stage were associated with USP8 expression (the $P$ values were 0.006 and 0.004, respectively). Regarding HER-3 expression, 


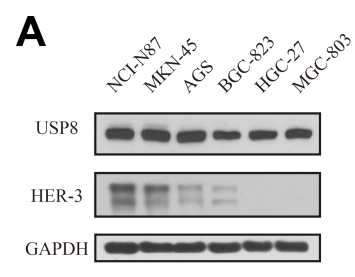

C
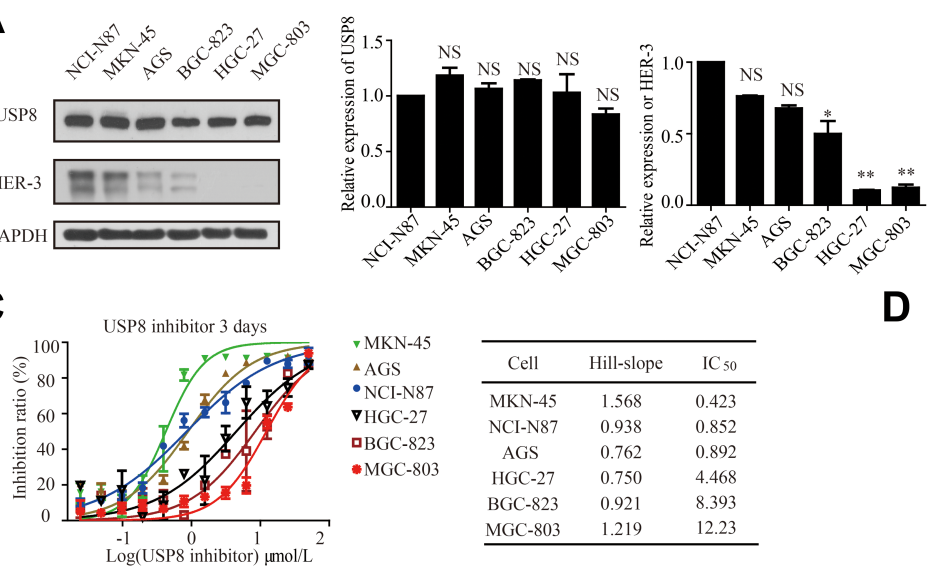

B

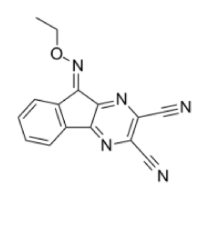

D

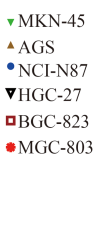

\begin{tabular}{ccc}
\hline Cell & Hill-slope & IC $_{50}$ \\
\hline MKN-45 & 1.568 & 0.423 \\
NCI-N87 & 0.938 & 0.852 \\
AGS & 0.762 & 0.892 \\
HGC-27 & 0.750 & 4.468 \\
BGC-823 & 0.921 & 8.393 \\
MGC-803 & 1.219 & 12.23 \\
\hline
\end{tabular}

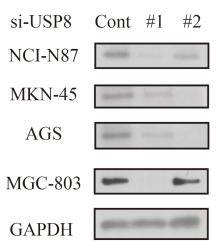

E
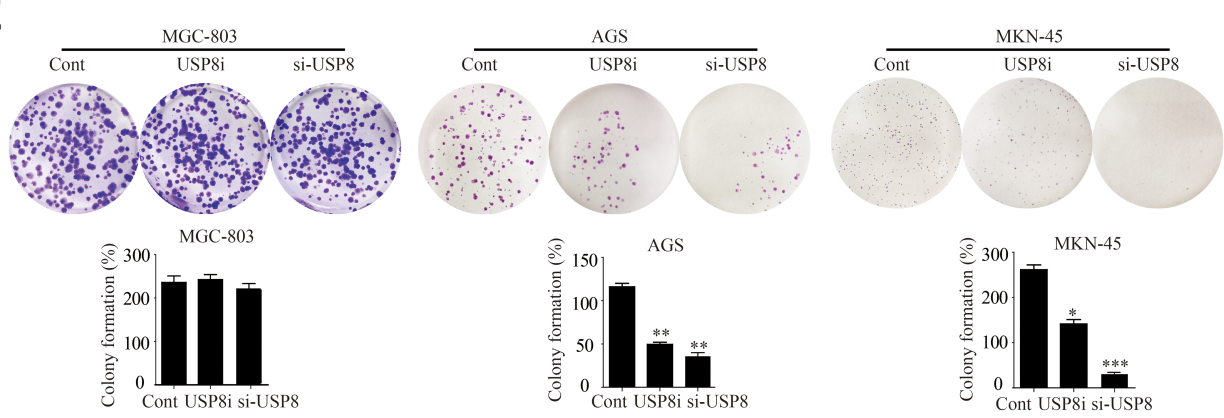

Figure 2 Down-regulation of USP8 inhibits proliferation of gastric cancer cells. (A) Expression levels of USP8 and HER-3 in six gastric cancer lines as indicated, $* P<0.05$, $* * P<0.01$. (B) Chemical structure of USP8 inhibitor (DUBs-IN-2). (C) The proliferation rates of cells were evaluated with indicated treatment. (D) Immunoblot analysis to confirm knockdown of USP8 using si-USP8 (si-USP8 sequence \#I and si-USP8 sequence \#2) and gene glyceraldehyde-3-phosphate dehydrogenase (GAPDH) was used as a control to verify equal loading of protein in NCl-N87, MKN-45, AGS and MGC-803 cell lines. (E) Clone formation experiments were performed on MGC-803 (USP8i, $2000 \mathrm{nmol} / \mathrm{L}$ and si-USP8 sequence \#I), MKN-45 (USP8i, $500 \mathrm{nmol} / \mathrm{L}$ and si-USP8 sequence \#I) and AGS (USP8i, $500 \mathrm{nmol} / \mathrm{L}$ and si-USP8 sequence $\# \mathrm{I}$ ) cells, $* P<0.05$, $* * P<0.0 \mathrm{I}$, $* * * P<0.00 \mathrm{I}$.

Abbreviations: NS, no statistical significance; USP8i, USP8 inhibitor.

statistically significant differences existed in nerve invasion, N stage, and pTNM stage (the $P$ values were $0.016,0.001$ and 0.021 , respectively). The Kaplan-Meier plot in Figure $1 \mathrm{~F}$ showed that patients with high USP8 expression had a much shorter survival time than the low cases $(P=0.009)$, and the same tendency was present in HER-3 $(P=0.001)$ in Figure 1G. What's more, the greatest difference in survival was found between patients with both markers being high expression and those with the opposite pattern (both markers low expression) (Figure 1H). Moreover, the expression of USP8 and HER-3 was closely correlated in gastric cancers $\left(\mathrm{R}^{2}=0.640, P<0.001\right)$ (Table 2 , Figure 1I), which manifested that the combined analysis of the two proteins might be more valuable in predicting the survival for GC.

We than estimated the relative risks (RR), both univariate and multivariate, of dying and $95 \% \mathrm{CI}$, using the Cox proportional hazard model in Table 3 . The Cox model shown that high expressions of USP8 and HER3, tumor size, nerve invasion, vascular tumor emboli as well as high grades of T, $\mathrm{N}, \mathrm{M}$, and pTNM stages were predictors of a poor prognosis in the univariate analysis. Then, the variables with statistical values of $P<0.05$ were selected for multivariate analysis, and USP8 and HER-3 retained their significance after adjustments for other known prognostic factors, which revealed that high expression of USP8 and HER-3 was unfavorable independent prognostic factors $(P=0.037$ and $P=0.003$, respectively) for GC patients.

\section{Down-Regulation of USP8 Inhibits Proliferation of Gastric Cancer Cells}

To investigate the role of USP8 and HER-3 in GC progression, their expressions were evaluated in six GC cell lines, including NCI-N87, MKN-45, AGS, BGC-823, HGC-27 and MGC-803. However, HER-3 was obvious overexpressed in NCI-N87, MKN-45 and AGS (Figure 2A). Thus, MGC803 was used as control as HER-3 was absent and NCI-N87, MKN-45 and AGS were selected as the HER-3 positive experimental group. In order to find out whether the pharmacological inactivation of USP8 could inhibit the growth of GC cells and was related to HER-3, USP8 inhibitor 
A

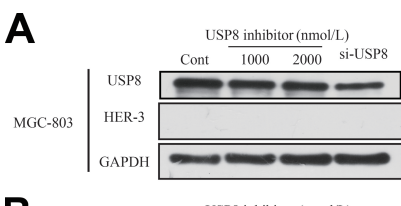

B

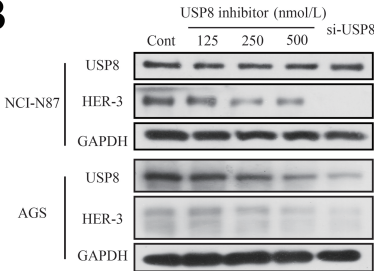

C
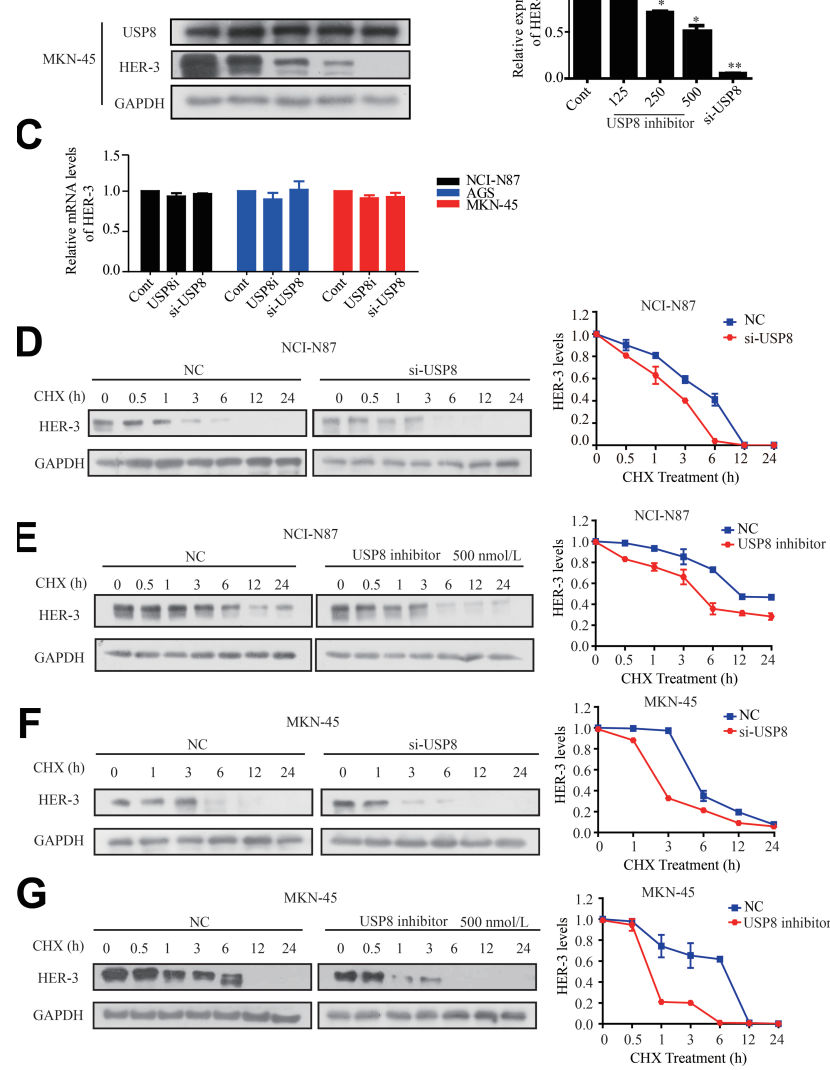

Figure 3 Down-regulation of USP8 promotes the degradation of HER-3. (A) Different concentrations of USP8 inhibitor in MGC-803 and si-USP8 cells were used as the control group. (B) Different concentrations of USP8 inhibitor and siUSP8 in NCI-N87, MKN-45 and AGS cells. (C) mRNA level of HER-3 in NCl-N87, AGS and MKN-45 cell lines with USP8 inhibitor and si-USP8 treatment. Expression of HER-3 in NCI-N87 cells with the treatment of USP8 inhibitor (D) and si-USP8 (E) and cycloheximide $(\mathrm{CHX}, 20 \mu \mathrm{g} / \mathrm{mL})$ in combination or alone. Expression of HER-3 in MKN-45 cells with the treatment of USP8 inhibitor (F) and si-USP8 (G) and $\mathrm{CHX}(20 \mu \mathrm{g} / \mathrm{mL})$ in combination or alone. $* P<0.05$, $* * P<0.01$.

Abbreviation: NS, no statistical significance.

(DUBs-IN-2, Figure 2B) was applied to evaluate the antiproliferation activity in these cell lines in Figure 2C. The result indicated that the antiproliferation effect of USP8 inhibitor on NCI-N87, MKN-45 and AGS was significantly higher than that of MGC-803, HGC-27 and BGC-823 (Figure 2C). Subsequently, we transfected the four selected gastric cancer cell lines with small interfering RNA of USP8, as shown in Figure 2D, and the subsequent si-usp8 were all carried out with \#1. Then, we performed a clonal formation experiment in Figure 2E to verify the results and found that USP8 inhibitor could significantly inhibit the proliferation of MKN-45 and AGS (HER-3 positive GC cells) compared with MGC-803 (HER-3 negative GC cells). Therefore, it was confirmed that down-regulation of USP8 could inhibit the proliferation of NCI-N87, MKN-45 and AGS cell lines, which is HER-3 positive GC cells.

\section{Down-Regulation of USP8 Promotes the Degradation of HER-3}

To investigate the effect of USP8 inhibitor on the expression of HER-3, different concentrations of USP8 inhibitor and si-USP8 were added in NCI-N87, MKN-45 and AGS cells, and MGC-803 as the control group (Figure 3A). It indicated that the expression of HER-3 decreased gradually with the increasing concentration of USP8 inhibitor and si-USP8, respectively, in NCI-N87, MKN-45 and AGS (Figure 3B). In addition, the HER-3 mRNA level was not affected by them in these three cell lines (Figure 3C), which manifested that downregulation of USP8 might regulate the stability of HER-3. So, NCI-N87 and MKN-45 AGS cells were treated with USP8 inhibitor and si-USP8 in the presence of cycloheximide (CHX, an inhibitor of protein synthesis). As shown in Figure 3DG, at 6-hour posttreatment, HER-3 protein was almost completely degraded in NCI-N87 and MKN-45 cells compared with their control groups. These results indicated that down-regulation of USP8 shortens the half-life of HER3 , and USP8 inhibitor might stabilize HER-3 by reducing the degradation of HER-3 protein, then inhibited the proliferation of GC cells.

\section{Down-Regulation of USP8 Inhibitor Affects the Cell-Cycle and Apoptosis of HER-3 Positive GC Cells}

At present, there is no research on the effect of USP8 inhibitors on tumor cell-cycle and apoptosis. Therefore, we found that down-regulation of USP8 could significantly inhibit the cell-cycle of AGS and block the G1 phase. But the MGC is not affected (Figure 4A). Then we performed the apoptosis experiment of USP8 on gastric cancer cells. The results indicated that the down-regulation of USP8 could significantly promote 

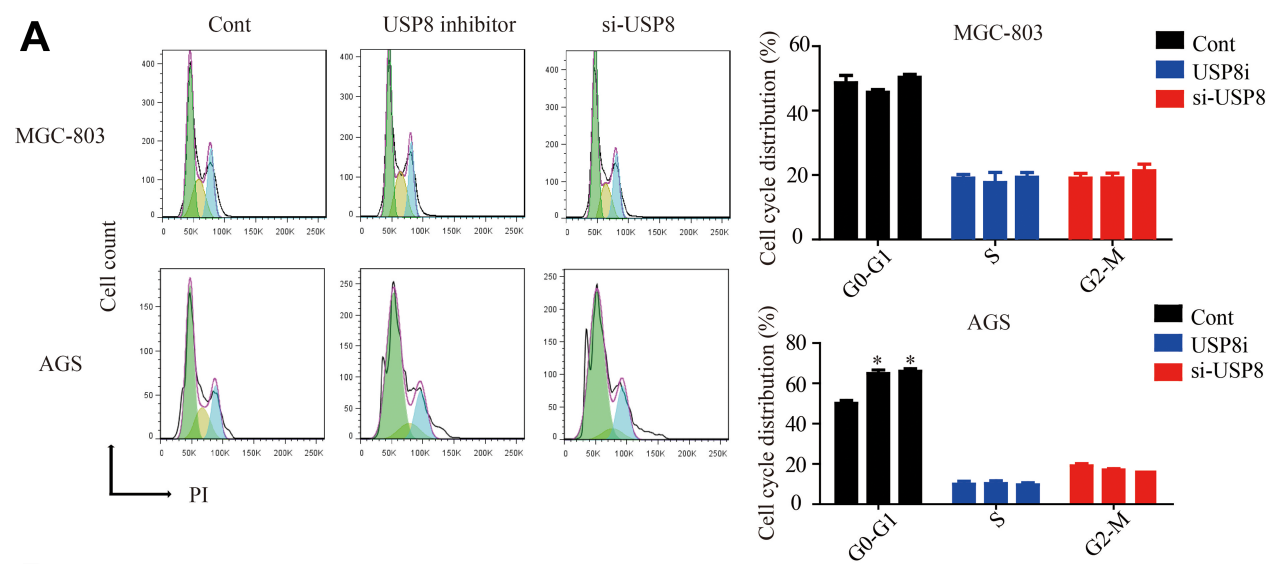

B
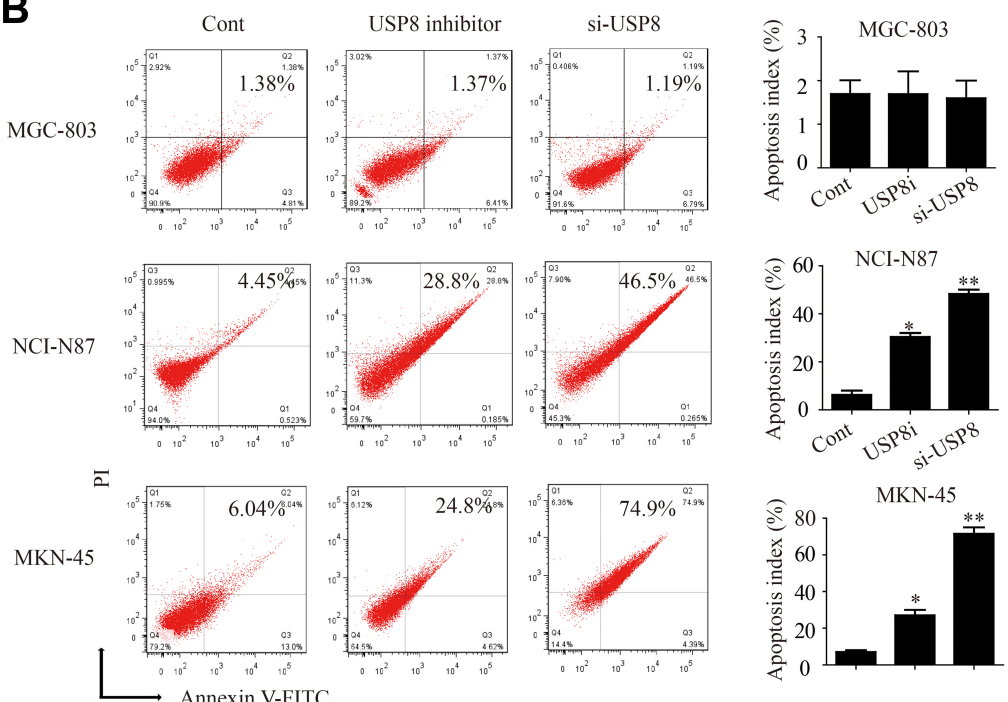

Figure 4 Down-regulation of USP8 affects the cell-cycle and apoptosis of HER-3 positive gastric cancer cells. (A) Cell-cycle experiments were performed on MGC-803 and AGS cells. (B) Apoptosis experiments were performed on MGC-803, NCl-N87 and MKN-45 cells. $* P<0.05, * * P<0.01$.

the apoptosis of NCI-N87 and MKN-45 cells, but it did not work on MGC-803 cells (Figure 4B). These results indicated that down-regulation of USP8 could promote the apoptosis of HER3-positive GC cells and inhibit the proliferation of them by affecting the cellcycle.

\section{Down Regulation of USP8 Inhibits GC Tumors Growth in Xenograft Model}

To explore the anti-cancer activity of USP8 in vivo, NCI-N87, MKN-45 and MGC-803 cells were subcutaneously transplanted into nude mice. The results of Figure 5A of the MGC-803 group showed that the tumor size, body weight and tumor weight of mice in each group did not change significantly. However, in NCI-N87 and MKN-45 groups indicated that the tumor growth of mice in inhibitor and si-USP8 groups were significantly reduced compared with the blank control group, as well as the tumor weight. And the effect of the two cell lines on tumor was not significantly different between the USP8 inhibitor and si-USP8 group. In addition, the growth of mice in each group was not significantly affected (Figure 5B and C). To further study the effect of down-regulation of USP8 on the proliferation of gastric cancer, expression of HER-3 and Ki-67 was examined in MGC-803, NCI-N87 and MKN-45 bearing mice, and results indicated that the expression of HER-3 and Ki-67 in USP8 inhibitor and si-USP8 groups in NCIN87 and MKN-45 were significantly lower than that in the control group, while there was no significant change in the expression of HER-3 and Ki-67 in MGC-803 group, specifically, HER-3 was absent in tumor from mice bearing MGC-803 cells (Figure 5D). All these results indicated that down-regulation of USP8 could inhibit the proliferation of HER-3 positive cells, NCIN87 and MKN-45, in vivo. 
A
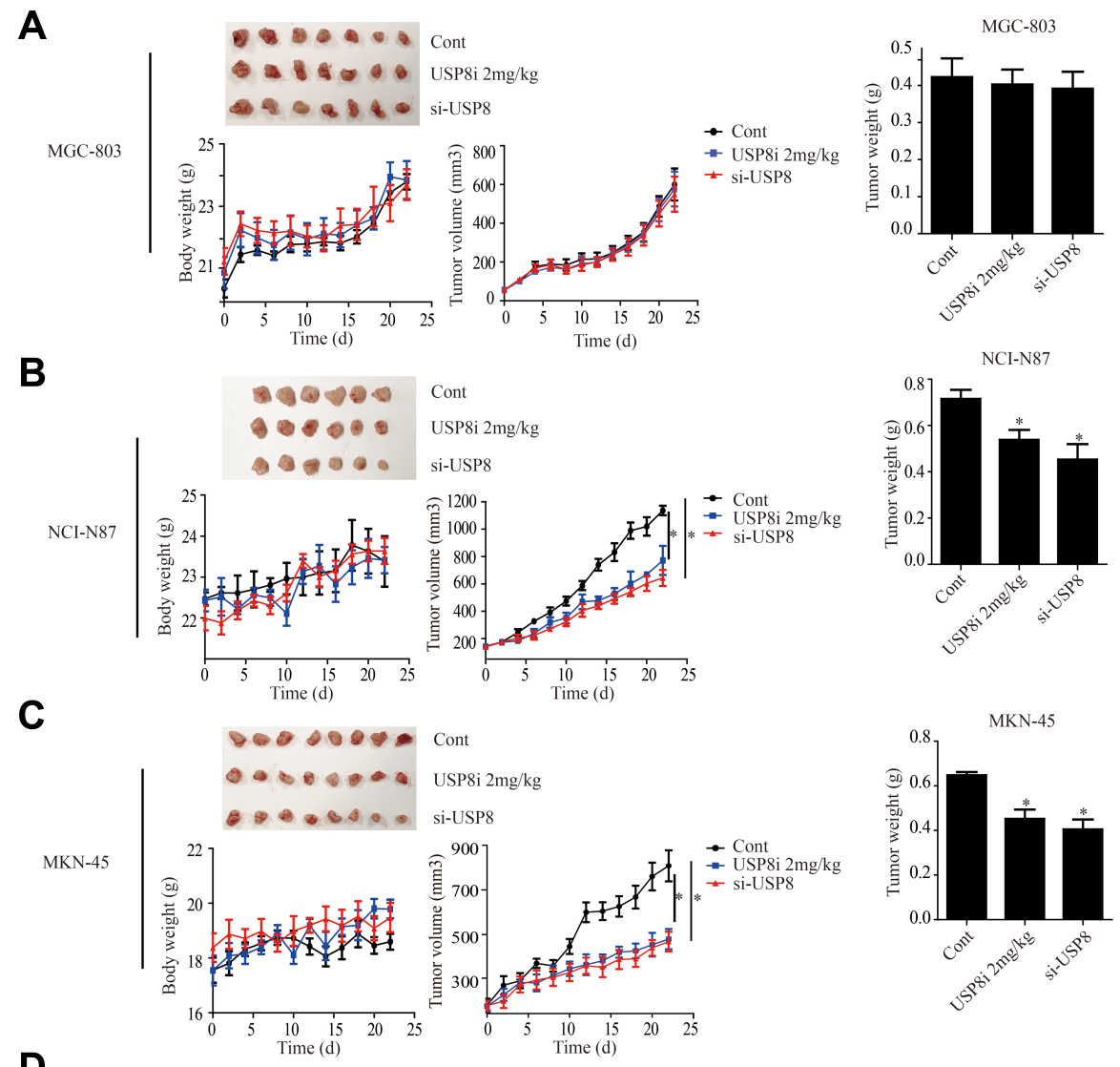

D
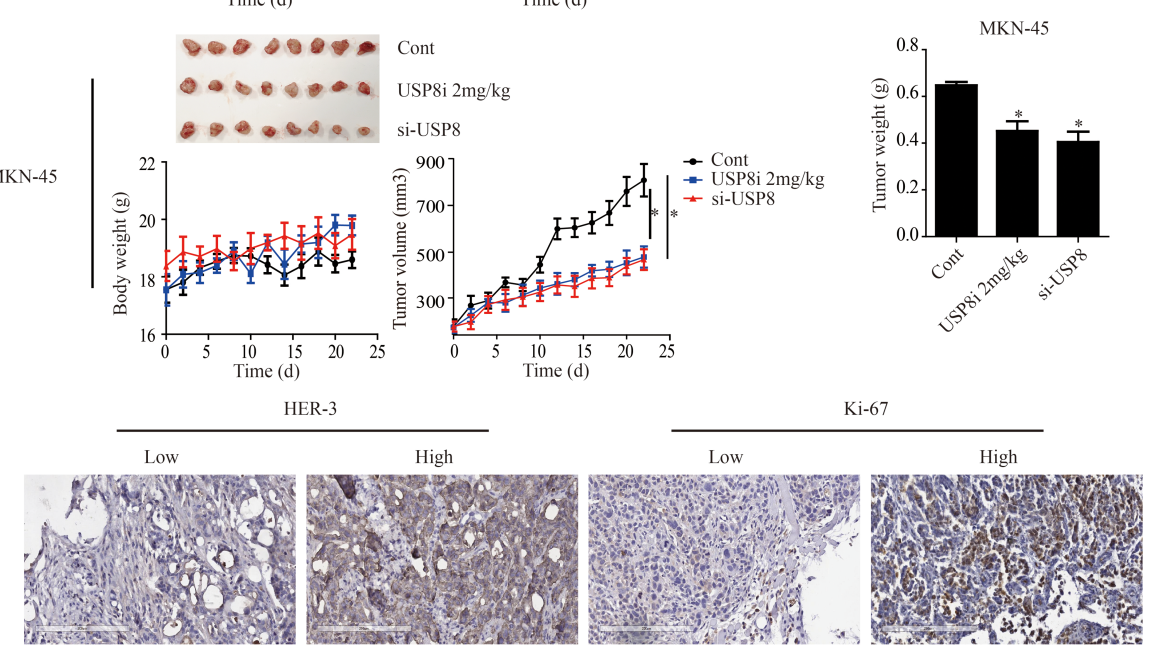
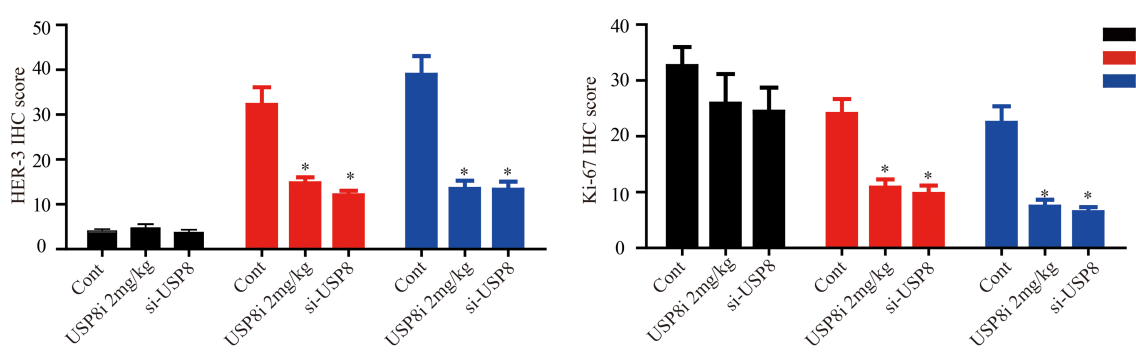

Figure 5 USP8 inhibitor inhibits gastric cancer tumors growth in xenograft model. (A) USP8 inhibitor and si-USP8 cells were injected in MGC-803 group, which used as the control group. USP8 inhibitor and si-USP8 in NCl-N87 (B) and MKN-45 (C) cells. Bar graph represents the results of the average tumor weight (presented as the mean \pm standard deviation). ${ }^{* P}<0.05$. (D) HER-3 and Ki-67 expression levels in tumor from mice bearing MGC-803, NCl-N87 and MKN-45 with USP8 inhibitor and si-USP8 treatment, and immunohistochemical staining (IHC) expression of Ki-67 and HER-3 in them. $* P<0.05$.

\section{Discussion}

Molecular markers associated with different clinical outcomes in GC have been identified, laying the foundation for improved clinical management through more personalized drugs. ${ }^{26}$ Despite improvements in recent years, the prognosis for gastric cancer is still unsatisfactory. Considerable evidence now exists that alteration in the expression of USP8 or HER-3 may serve as an important pathogenic event for some human malignancies. ${ }^{27,28}$ However, the effect and mechanism of USP8 and HER-3 on GC is still unrevealed.

In the present study, we found that the expression of USP8 and HER-3 was significantly upregulated in GC tissues compared with adjacent control samples. We also 
found that high expression of USP8 and HER-3 predicted poor prognosis of GC patients. And increased USP8 and HER-3 protein expression correlated with poor survival, suggesting that USP8 and HER-3 are prospective biomarkers for GC diagnosis and therapy, although more work still needed to be done.

Then, effect of USP8 and HER-3 on the biological behavior of GC cells was explored. Reduced proliferation was observed in both in vitro and in vivo cells treated with USP8 inhibitor and si-USP8. All in vitro results demonstrated that down-regulation of USP8 inhibited the proliferation and viability of GC cells with high expression of HER3 (NCI-N87, MKN-45 and AGS), but did not affect HER3negative cells (MGC-803). In vivo, compared with those of tumors from the control group (MGC-803), the volumes and weights of tumors from HER-3-positive groups (NCI-N87 and $\mathrm{MKN}-45$ ) were significantly decreased.

However, the research team will continue to explore the mechanism of USP8 on HER-3 in GC. Some of the literature suggests that HER-3 overexpression may be associated with poor prognosis and unfavorable survival mediated by PI3K/AKT signaling pathway. ${ }^{21}$ As known to all, $\mathrm{PI} 3 \mathrm{~K} / \mathrm{AKT}$ is a common signaling pathway downregulated in gastric cancer. ${ }^{29,30}$ Therefore, it can be inferred that down-regulation of USP8 may inhibit the proliferation and even metastasis of GC through this pathway. Other studies have suggested that mutations in USP8 reduce the degradation of EGFR, such as HER-2 and HER-3, thereby promoting tumor progression. ${ }^{14,31} \mathrm{So}$, what is the situation in gastric cancer?

\section{Conclusion}

Down-regulation of USP8 inhibits HER-3 positive GC cells proliferation in vivo and in vitro, which indicate that USP8 represents a feasible choice as a therapeutic target for HER-3 positive GC cells.

\section{Abbreviations}

AJCC, American Cancer Society; DUBs, deubiquitinating enzymes; EGF, epidermal growth factor; EGFR, epidermal growth factor receptor; GC, gastric cancer; GEPIA, gene expression profiling interactive analysis; HER-2, human epidermal growth factor receptor 2; HER-3, human epidermal growth factor receptor-3; IHC, immunohistochemical staining; HE, hematoxylin-eosin staining; PCR, polymerase chain reactions; PI3K/AKT, phosphatidylinositol-3-kinases/protein-serine-threonine kinase; qRT-PCR, quantitative real-time PCR; RR, relative risks; RTK, receptor tyrosine kinases; USP8i, USP8 inhibitor; siRNA, small interfering RNA; TNM, tumor node metastasis; USP8, ubiquitin specific peptidase 8; UICC, International Union Against Cancer; WHO, World Health Organization.

\section{Ethical Approval}

All animal experiments as described above were approved by the Institutional Animal Care and Use Committee of Zhengzhou University and performed based on the guidelines of the Institutional Animal Care and Use Committee of Zhengzhou University. The study with human samples was approved by the Ethics Committee of the First Affiliated Hospital of Zhengzhou University. And we clarify that all clinical samples described here were gained from patients who had given written informed consent.

\section{Author Contributions}

All authors made a significant contribution to the work reported, whether that is in the conception, study design, execution, acquisition of data, analysis and interpretation, or in all these areas; took part in drafting, revising or critically reviewing the article; gave final approval of the version to be published; have agreed on the journal to which the article has been submitted; and agree to be accountable for all aspects of the work.

\section{Funding}

This work was supported by the National Natural Science Foundation of China (No.81602961, No. 81,430,085 and No.21372206).

\section{Disclosure}

The authors report no conflicts of interest in this work.

\section{References}

1. Bray F, Ferlay J, Soerjomataram I, Siegel RL, Torre LA, Jemal A. Global cancer statistics 2018: GLOBOCAN estimates of incidence and mortality worldwide for 36 cancers in 185 countries. CA Cancer J Clin. 2018;68(6):394-424. doi:10.3322/caac.21492

2. Torre LA, Bray F, Siegel RL, Ferlay J, Lortet-Tieulent J, Jemal A. Global cancer statistics, 2012. CA Cancer J Clin. 2015;65(2):87-108. doi: $10.3322 /$ caac. 21262

3. Collaborators GBDSC. The global, regional, and national burden of stomach cancer in 195 countries, 19902017: a systematic analysis for the global burden of disease study 2017. Lancet Gastroenterol Hepatol. 2020;5(1):42-54. doi:10.1016/S2468-1253(19)30328-0.

4. Ajani JA, Lee J, Sano T, Janjigian YY, Fan D, Song S. Gastric adenocarcinoma. Nat Rev Dis Primers. 2017;3:17036. doi:10.1038/ $\operatorname{nrdp} .2017 .36$ 
5. DeSantis $\mathrm{CE}$, Lin $\mathrm{CC}$, Mariotto $\mathrm{AB}$, et al. Cancer treatment and survivorship statistics, 2014. CA Cancer J Clin. 2014;64 (4):252-271. doi:10.3322/caac. 21235

6. Jie M, Wu Y, Gao M, et al. CircMRPS35 suppresses gastric cancer progression via recruiting KAT7 to govern histone modification. Mol Cancer. 2020;19(1):56. doi:10.1186/s12943-020-01160-2

7. Zhang $\mathrm{E}$, He $\mathrm{X}$, Zhang $\mathrm{C}$, et al. A novel long noncoding RNA HOXC-AS3 mediates tumorigenesis of gastric cancer by binding to YBX1. Genome Biol. 2018;19(1):154. doi:10.1186/s13059-0181523-0

8. Lin JX, Xie XS, Weng XF, et al. UFM1 suppresses invasive activities of gastric cancer cells by attenuating the expres7sion of PDK1 through PI3K/AKT signaling. J Exp Clin Cancer Res. 2019;38 (1):410. doi:10.1186/s13046-019-1416-4

9. Kuai X, Li L, Chen R, et al. SCF(FBXW7)/GSK3beta-Mediated GFI1 degradation suppresses proliferation of gastric cancer cells. Cancer Res. 2019;79(17):4387-4398. doi:10.1158/0008-5472.CAN$18-4032$

10. Neou M, Villa C, Armignacco R, et al. Pangenomic classification of pituitary neuroendocrine tumors. Cancer Cell. 2020;37(1):123-134 e125. doi:10.1016/j.ccell.2019.11.002

11. McHugh A, Fernandes K, Chinner N, et al. The identification of potential therapeutic targets for cutaneous squamous cell carcinoma. $J$ Invest Dermatol. 2020;140(6):1154-1165 e1155. doi:10.1016/j. jid.2019.09.024

12. Shin S, Kim K, Kim HR, et al. Deubiquitylation and stabilization of Notch1 intracellular domain by ubiquitin-specific protease 8 enhance tumorigenesis in breast cancer. Cell Death Differ. 2020;27 (4):1341-1354. doi:10.1038/s41418-019-0419-1

13. Sun J, Hu Q, Peng H, et al. The ubiquitin-specific protease USP8 deubiquitinates and stabilizes Cx43. J Biol Chem. 2018;293 (21):8275-8284. doi:10.1074/jbc.RA117.001315

14. Kim Y, Shiba-Ishii A, Nakagawa T, et al. Stratifin regulates stabilization of receptor tyrosine kinases via interaction with ubiquitin-specific protease 8 in lung adenocarcinoma. Oncogene. 2018;37(40):5387-5402. doi:10.1038/s41388-018-0342-9

15. Hua X, Huang M, Deng X, et al. The inhibitory effect of compound ChlA-F on human bladder cancer cell invasion can be attributed to its blockage of SOX2 protein. Cell Death Differ. 2020;27(2):632-645. doi:10.1038/s41418-019-0377-7

16. Jeong M, Lee EW, Seong D, et al. USP8 suppresses death receptor-mediated apoptosis by enhancing FLIPL stability. Oncogene. 2017;36(4):458-470. doi:10.1038/onc.2016.215

17. Dufner A, Knobeloch KP. Ubiquitin-specific protease 8 (USP8/ UBPy): a prototypic multidomain deubiquitinating enzyme with pleiotropic functions. Biochem Soc Trans. 2019;47(6):1867-1879. doi:10.1042/BST20190527

18. Ma ZY, Song ZJ, Chen JH, et al. Recurrent gain-of-function USP8 mutations in Cushing's disease. Cell Res. 2015;25(3):306-317. doi:10.1038/cr.2015.20
19. Reincke M, Sbiera S, Hayakawa A, et al. Mutations in the deubiquitinase gene USP8 cause Cushing's disease. Nat Genet. 2015;47 (1):31-38. doi:10.1038/ng.3166

20. Byun S, Lee SY, Lee $\mathrm{J}$, et al. USP8 is a novel target for overcoming gefitinib resistance in lung cancer. Clin Cancer Res. 2013;19 (14):3894-3904. doi:10.1158/1078-0432.CCR-12-3696

21. Wang L, Yuan H, Li Y, Han Y. The role of HER3 in gastric cancer. Biomed Pharmacother. 2014;68(6):809-812. doi:10.1016/j.biopha.20 14.08.011

22. Zhao J, Bi W, Zhang J, et al. USP8 protects against lipopolysaccharide-induced cognitive and motor deficits by modulating microglia phenotypes through TLR4/MyD88/NF-kappaB signaling pathway in mice. Brain Behav Immun. 2020. doi:10.1016/j. bbi.2020.04.052

23. Nagtegaal ID, Odze RD, Klimstra D, et al. The 2019 WHO classification of tumours of the digestive system. Histopathology. 2020;76 (2):182-188. doi:10.1111/his.13975

24. In H, Solsky I, Palis B, Langdon-Embry M, Ajani J, Sano T. Validation of the 8th edition of the AJCC TNM staging system for gastric cancer using the national cancer database. Ann Surg Oncol. 2017;24(12):3683-3691. doi:10.1245/s10434-017-6078-x

25. Tang Z, Li C, Kang B, Gao G, Li C, Zhang Z. GEPIA: a web server for cancer and normal gene expression profiling and interactive analyses. Nucleic Acids Res. 2017;45(W1):W98W102. doi:10.1093/ nar/gkx247

26. Oh DY, Bang YJ. HER2-targeted therapies - a role beyond breast cancer. Nat Rev Clin Oncol. 2020;17(1):33-48. doi:10.1038/s41571019-0268-3

27. Cao Z, Wu X, Yen L, Sweeney C, Carraway KL 3rd. Neuregulininduced ErbB3 downregulation is mediated by a protein stability cascade involving the E3 ubiquitin ligase Nrdp1. Mol Cell Biol. 2007;27(6):2180-2188. doi:10.1128/MCB.01245-06

28. Le Clorennec C, Lazrek Y, Dubreuil O, et al. ITCH-dependent proteasomal degradation of c-FLIP induced by the anti-HER3 antibody 9F7-F11 promotes DR5/caspase 8-mediated apoptosis of tumor cells. Cell Commun Signal. 2019;17(1):106. doi:10.1186/s12964-0190413-8

29. Huang Z, Liang H, Chen L. RAB43 promotes gastric cancer cell proliferation and metastasis via regulating the PI3K/AKT signaling pathway. Onco Targets Ther. 2020;13:2193-2202. doi:10.2147/OTT. S237356

30. Mayer IA, Arteaga CL. The PI3K/AKT pathway as a target for cancer treatment. Annu Rev Med. 2016;67:11-28. doi:10.1146/ annurev-med-062913-051343

31. Bujko M, Kober P, Boresowicz J, et al. USP8 mutations in corticotroph adenomas determine a distinct gene expression profile irrespective of functional tumour status. Eur J Endocrinol. 2019;181 (6):615-627. doi:10.1530/EJE-19-0194
OncoTargets and Therapy

\section{Publish your work in this journal}

OncoTargets and Therapy is an international, peer-reviewed, open access journal focusing on the pathological basis of all cancers, potential targets for therapy and treatment protocols employed to improve the management of cancer patients. The journal also focuses on the impact of management programs and new therapeutic agents and protocols on patient perspectives such as quality of life, adherence and satisfaction. The manuscript management system is completely online and includes a very quick and fair peer-review system, which is all easy to use. Visit http://www.dovepress.com/ testimonials.php to read real quotes from published authors. 